\title{
The Reaction of Phenyl Iso(thio)cyanate with N-Heterocyclic Carbene-Supported Nickel Complexes: Formation of Nickelacycles
}

\author{
Stefan Pelties and Robert Wolf*
}

Institute of Inorganic Chemistry, University of Regensburg, 93040 Regensburg, Germany

The supporting information contains:

(S1) ${ }^{1} \mathrm{H}$ and ${ }^{13} \mathrm{C}\left\{{ }^{1} \mathrm{H}\right\}$ NMR spectra of 1 and 3

(S2) X-ray crystallography 


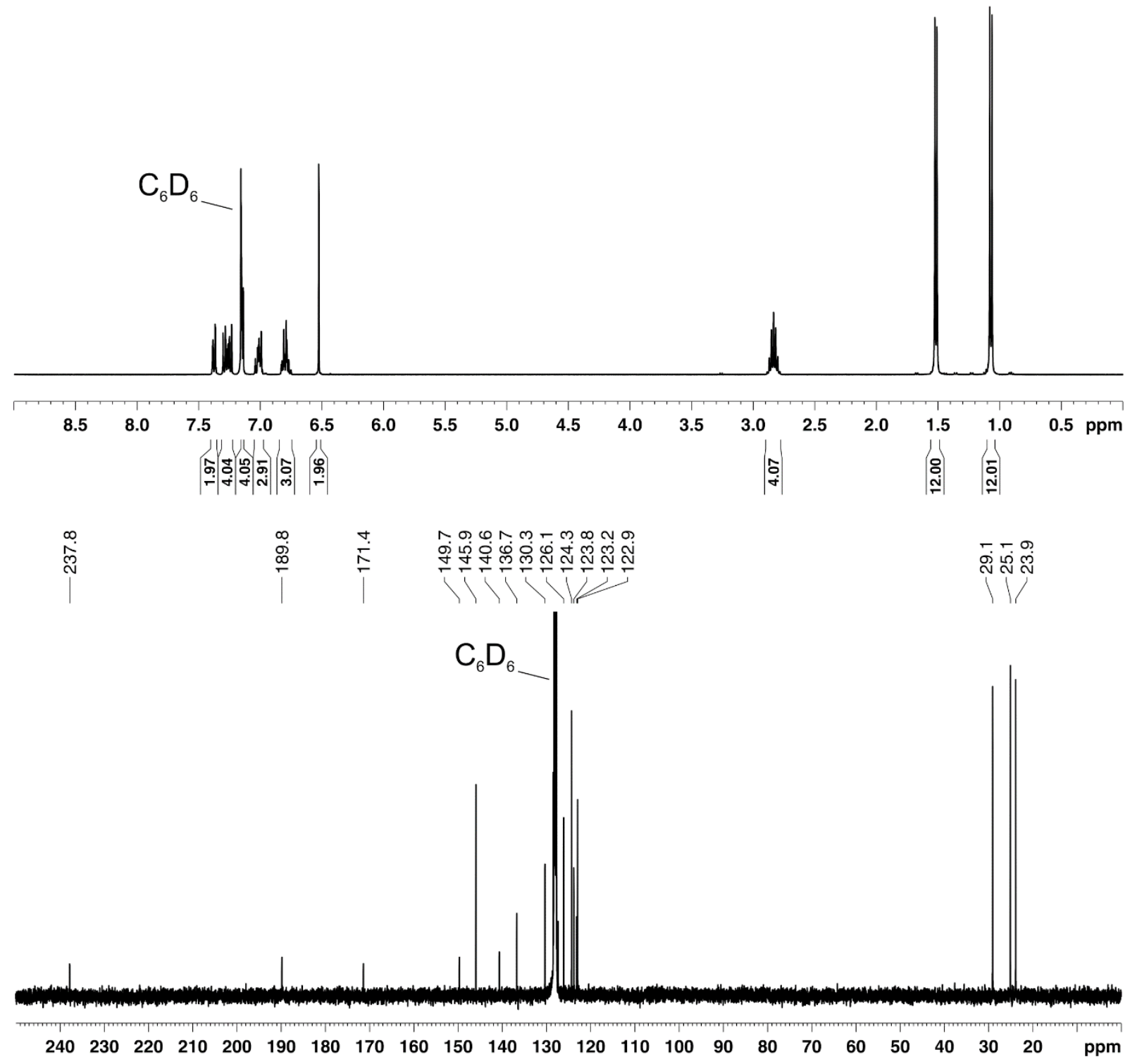

Figure S1. ${ }^{1} \mathrm{H}$ (top) and ${ }^{13} \mathrm{C}\left\{{ }^{1} \mathrm{H}\right\}$ NMR spectra (bottom) of $1\left(400.16 / 100.61 \mathrm{MHz}, \mathrm{C}_{6} \mathrm{D}_{6}, 300 \mathrm{~K}\right)$. 


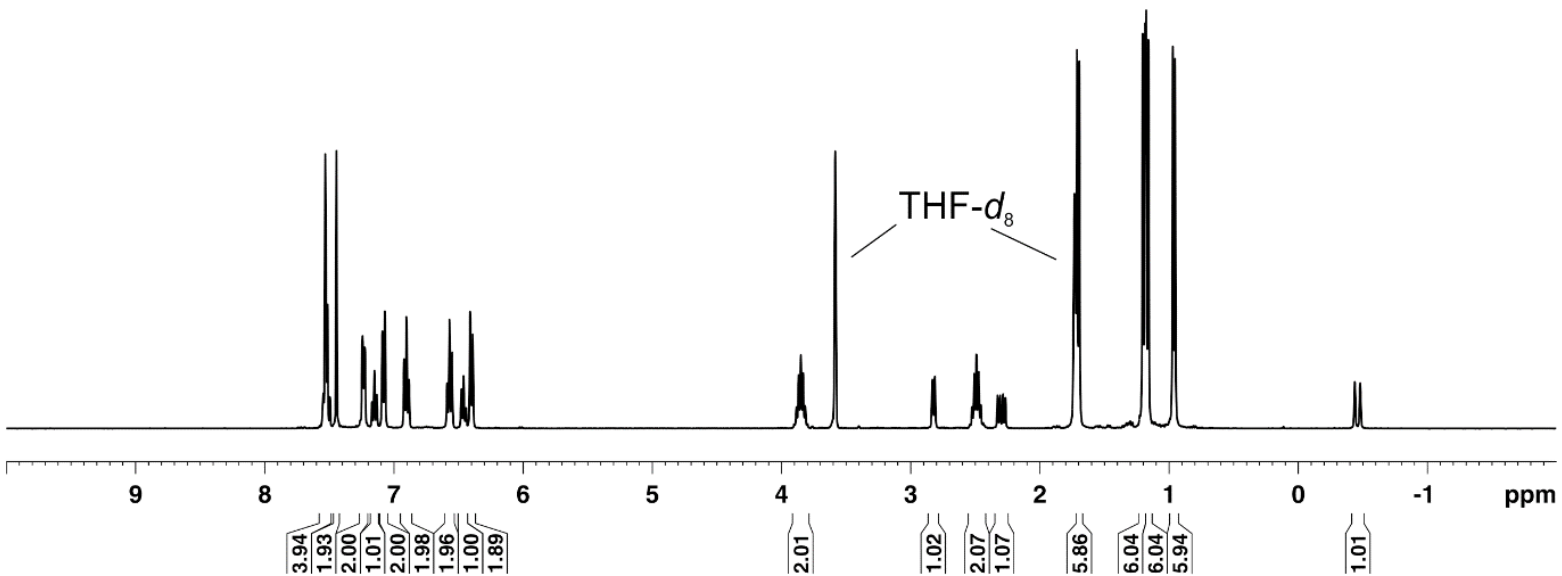

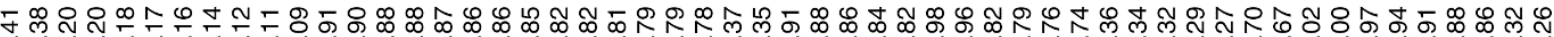

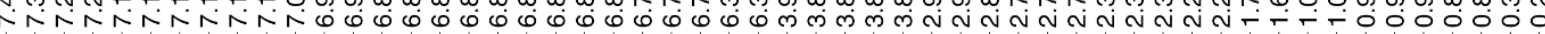

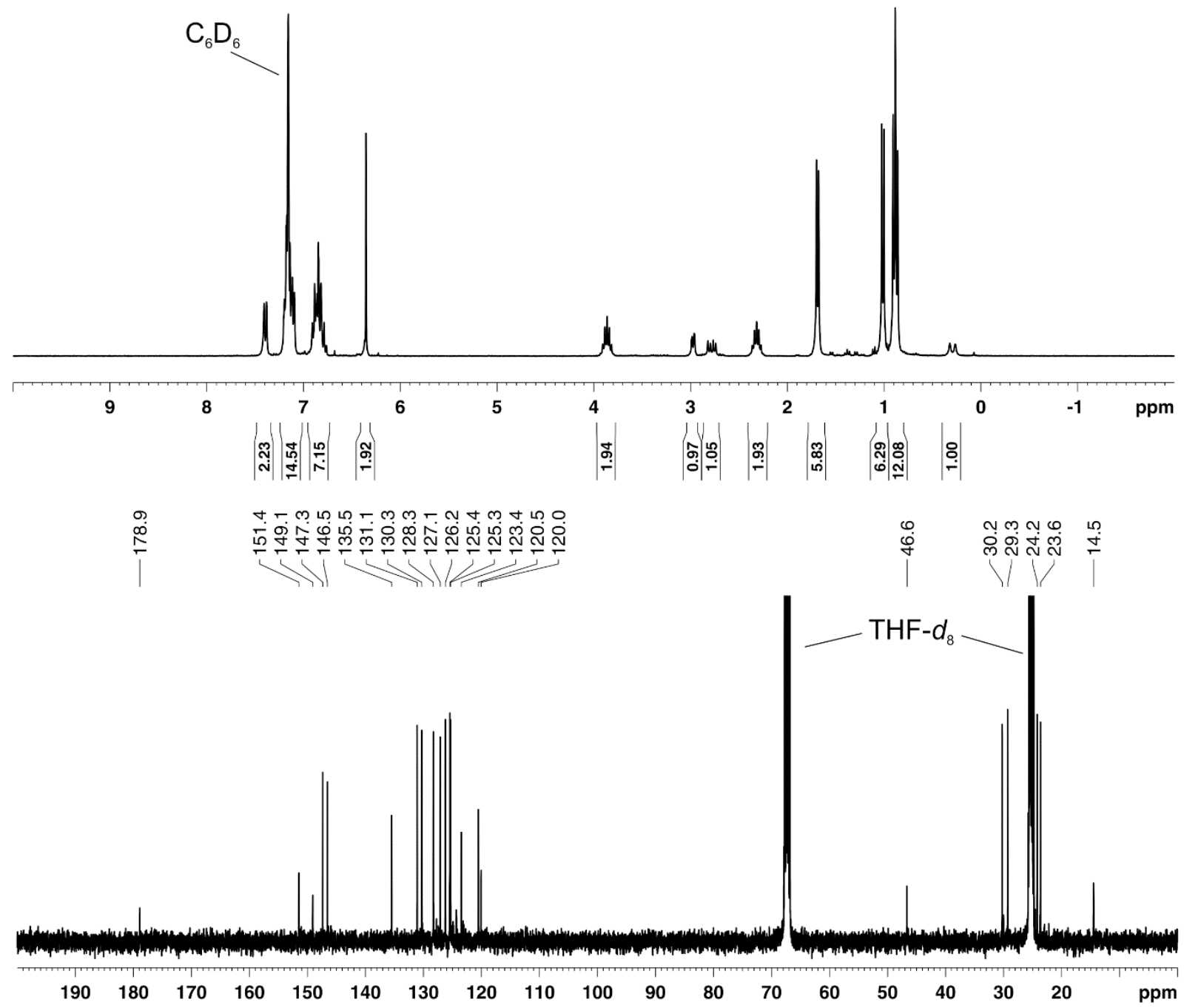

Figure S2. ${ }^{1} \mathrm{H}$ (top, THF- $\left.d_{8}\right),{ }^{1} \mathrm{H}\left(\right.$ middle, $\left.\mathrm{C}_{6} \mathrm{D}_{6}\right)$ and ${ }^{13} \mathrm{C}\left\{{ }^{1} \mathrm{H}\right\}$ NMR spectra (bottom, THF- $d_{8}$ ) of 3 (400.16/400.16/100.61 MHz, 300 K). 


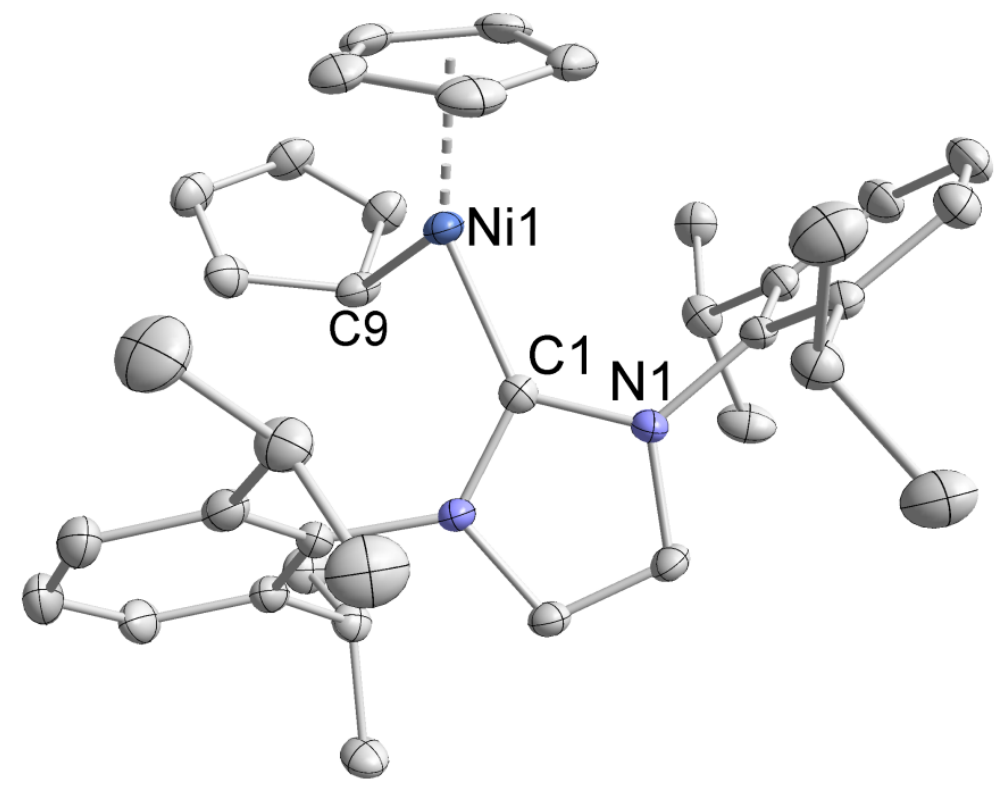

Figure S3. Solid-state molecular structure of $\left[\left(\eta^{5}-\mathrm{Cp}\right) \mathrm{Ni}\left(\eta^{1}-\mathrm{Cp}\right)(\mathrm{IDipp})\right]$ (2). The hydrogen atoms are omitted for clarity. Thermal ellipsoids are drawn at $40 \%$ level. Selected bond length $[\AA]$ and angles $\left[^{\circ}\right]$ : Ni1-C1 1.881(2), Ni1-C9 2.021(3). 
Table S1. Crystallographic data of 1, 2, and 3

\begin{tabular}{|c|c|c|c|}
\hline Compound & 1 & 2 & 3 \\
\hline Empirical formula & $\mathrm{C}_{41} \mathrm{H}_{46} \mathrm{~N}_{4} \mathrm{NiS}_{2}$ & $\mathrm{C}_{37} \mathrm{H}_{46} \mathrm{~N}_{2} \mathrm{Ni}$ & $\mathrm{C}_{42} \mathrm{H}_{49} \mathrm{~N}_{3} \mathrm{NiO}$ \\
\hline Formula weight & 717.65 & 577.47 & 670.55 \\
\hline Temperature $[\mathrm{K}]$ & $123(1)$ & $123(1)$ & $123(1)$ \\
\hline Crystal system & monoclinic & monoclinic & triclinic \\
\hline Space group & $C 2 / c$ & $P 2_{1} / n$ & $P-1$ \\
\hline $\mathrm{a}[\AA]$ & $21.2844(3)$ & $10.32832(18)$ & $10.3147(2)$ \\
\hline $\mathrm{b}[\AA]$ & $12.13298(17)$ & $19.6066(3)$ & $18.7357(5)$ \\
\hline $\mathrm{c}[\AA]$ & $29.3618(3)$ & $15.8877(3)$ & $19.4834(6)$ \\
\hline$\alpha\left[^{\circ}\right]$ & 90 & 90 & $84.582(2)$ \\
\hline$\beta\left[^{\circ}\right]$ & $92.0206(12)$ & $98.3793(15)$ & $82.009(2)$ \\
\hline$\gamma\left[{ }^{\circ}\right]$ & 90 & 90 & $89.3620(18)$ \\
\hline Volume $\left[\AA^{3}\right]$ & $7577.77(17)$ & $3182.98(9)$ & $3711.99(16)$ \\
\hline $\mathrm{Z}$ & 8 & 4 & 4 \\
\hline$\rho_{\text {calc }}\left[\mathrm{g} / \mathrm{cm}^{3}\right]$ & 1.258 & 1.205 & 1.200 \\
\hline$\mu\left[\mathrm{mm}^{-1}\right]$ & 2.017 & 1.063 & 1.012 \\
\hline $\mathrm{F}(000)$ & 3040.0 & 1240.0 & 1432.0 \\
\hline Crystal size $\left[\mathrm{mm}^{3}\right]$ & $0.8135 \times 0.3574 \times 0.1914$ & $0.3672 \times 0.3136 \times 0.2458$ & $0.4458 \times 0.1609 \times 0.0796$ \\
\hline Radiation & $\mathrm{CuK}_{\alpha}(\lambda=1.54184)$ & $\mathrm{CuK}_{\alpha}(\lambda=1.54184)$ & $\mathrm{CuK}_{\alpha}(\lambda=1.54184)$ \\
\hline $2 \Theta$ range for data collection $\left[{ }^{\circ}\right]$ & 8.314 to 133.274 & 7.208 to 133.502 & 6.908 to 133.442 \\
\hline Index ranges & $\begin{array}{l}-19 \leq \mathrm{h} \leq 25,-13 \leq \mathrm{k} \leq 14 \\
-34 \leq 1 \leq 34\end{array}$ & $\begin{array}{l}-11 \leq \mathrm{h} \leq 12,-22 \leq \mathrm{k} \leq 23 \\
-18 \leq 1 \leq 18\end{array}$ & $\begin{array}{l}-12 \leq \mathrm{h} \leq 12,-21 \leq \mathrm{k} \leq 22, \\
-23 \leq 1 \leq 23\end{array}$ \\
\hline Reflections collected & 23029 & 15944 & 47839 \\
\hline Independent reflections & $\begin{array}{l}6661\left[R_{\text {int }}=0.0418,\right. \\
\left.R_{\text {sigma }}=0.0372\right]\end{array}$ & $\begin{array}{l}5595\left[\mathrm{R}_{\text {int }}=0.0399,\right. \\
\left.\mathrm{R}_{\text {sigma }}=0.0372\right]\end{array}$ & $\begin{array}{l}13030\left[\mathrm{R}_{\text {int }}=0.0385,\right. \\
\left.\mathrm{R}_{\text {sigma }}=0.0336\right]\end{array}$ \\
\hline Data / restraints / parameters & $6661 / 0 / 441$ & $5595 / 0 / 369$ & $13030 / 0 / 867$ \\
\hline Goodness-of-fit on $\mathrm{F}^{2}$ & 1.123 & 1.144 & 1.114 \\
\hline Final $R$ indexes $[\mathrm{I}>=2 \sigma(\mathrm{I})]$ & $\mathrm{R}_{1}=0.0375, \mathrm{wR}_{2}=0.1033$ & $\mathrm{R}_{1}=0.0527, \mathrm{wR}_{2}=0.1417$ & $\mathrm{R}_{1}=0.0647, \mathrm{wR}_{2}=0.2327$ \\
\hline Final $\mathrm{R}$ indexes [all data] & $\mathrm{R}_{1}=0.0447, \mathrm{wR}_{2}=0.1163$ & $\mathrm{R}_{1}=0.0555, \mathrm{wR}_{2}=0.1438$ & $\mathrm{R}_{1}=0.0728, \mathrm{wR}_{2}=0.2371$ \\
\hline Largest diff. peak/hole $\left[\mathrm{e} \AA^{-3}\right.$ ] & $0.78 /-0.52$ & $0.84 /-0.59$ & $0.99 /-0.53$ \\
\hline
\end{tabular}

